\title{
ANÁLISIS
}

\section{EL INFORME FINCH Y EL ACCESO ABIERTO A LAS PUBLICACIONES BIOMEDICAS}

\section{The Finch Report and the open access to biomedical publications}

\section{Karim-Javier Gherab-Martín}

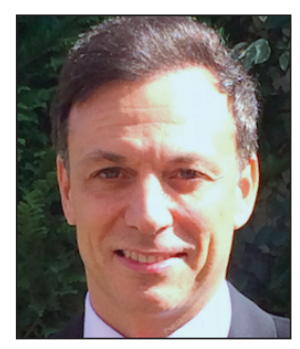

Karim-Javier Gherab-Martín, licenciado en física teórica y doctor en filosofía de la ciencia y la tecnología, es profesor en la Facultad de Humanidades y Ciencias de la Comunicación, de la Universidad CEU San Pablo. Ha sido profesor asociado en la Universidad Autónoma de Madrid y en la Universidad del País Vasco, así como investigador invitado y profesor asistente en la Harvard University (2008-2009) y en la University of Illinois Urbana-Champaign (2010). Ha realizado estancias de investigación en el CSIC (2011-2013) y en la Université Paris-Diderot (2013). Sus investigaciones y publicaciones recientes se han centrado en las implicaciones de la tecnología digital e internet en la ciencia, en la comprensión pública de la ciencia y la tecnología, y en las ideas filosóficas subyacentes en la comunicación y divulgación de la física.

http://orcid.org/0000-0003-2116-7347

Universidad CEU San Pablo Facultad de Humanidades y Ciencias de la Comunicación Paseo de Juan XIII, 6. 28040 Madrid, España karim.gherabmartin@ceu.es

kgmscholar@gmail.com

\section{Resumen}

Se analiza el origen de la bifurcación del movimiento open access en dos caminos: verde y dorado, y se explican las razones que han llevado al Reino Unido y a los EUA a optar por uno de esos caminos. El lobby a favor del camino dorado tiene sus orígenes en las políticas de publicación de la revista biomédica The New England journal of medicine: su director, Franz J. Ingelfinger, introdujo la norma de no aceptar para su publicación manuscritos que previamente hubieran circulado como pre-prints, norma que con el tiempo se ha dado en llamar "regla de Ingelfinger".

\section{Palabras clave}

Acceso abierto; ArXiv; Biomedicina; Informe Finch; Regla de Ingelfinger; Preprints; Tecnociencia.

\begin{abstract}
We analyse why the open access movement split into two paths, gold and green, and try to explain the reasons behind the choices between them that were made in the UK and USA. The analysis shows that lobbying in favor of the gold path had its origins in the publication policies of The New England journal of medicine: its director, Franz J. Ingelfinger, introduced the publication rule of not accepting manuscripts that previously had been circulating as pre-prints, known as the "Ingelfinger rule".
\end{abstract}

\section{Keywords}

ArXiv; Biomedicine; Finch report; Ingelfinger rule; Open access; Preprints; Technoscience.

Gherab-Martín, Karim-Javier (2015). "El Informe Finch y el acceso abierto a las publicaciones biomédicas". El profesional de la información, v. 24, n. 5, pp. 631-639. 


\section{Introducción. El Informe Finch}

Desde hace algunos años, muchos defensores del open access $(\mathrm{OA})^{1}$ están a favor de obligar a las revistas a poner en acceso abierto aquellos artículos cuyos resultados hayan sido financiados con fondos públicos. La idea fundamental que está detrás de este movimiento de OA es que la tecnología digital nos facilita recuperar de alguna manera el espíritu que acompañó los orígenes de la ciencia moderna: un diálogo entre científicos sin obstáculos ni intermediarios. El enorme desarrollo de la ciencia ha fomentado la presencia de editoriales comerciales que han sido capaces de promover, almacenar y distribuir la creciente masa de información académica, pero junto con sus innegables ventajas, este sistema de editoriales comerciales también ha creado un buen número de problemas. Uno de los mayores tiene que ver con el incremento de los precios de suscripción (Guédon, 2001).

Una serie de reuniones de los impulsores del OA ha dado lugar a una sucesión de declaraciones públicas ${ }^{2}$ y de recomendaciones que han establecido ciertas pautas en las iniciativas OA hasta el momento. Así, se identificaron ${ }^{3}$ dos caminos para alcanzar un OA completo: la vía dorada y la vía verde. Una descripción completa de la historia y evolución del movimiento OA puede leerse en Suber (2012a).

Como es sabido, la vía verde apela al auto-archivo, es decir, que los investigadores depositen sus artículos en repositorios digitales institucionales o temáticos, ya sean universitarios o de centros de investigación. En este caso, los autores suben sus preprints (textos que aún no han sido revisados por los pares) o postprints (textos que sí han sido revisados por los pares) al repositorio digital como paso final de sus investigaciones. Sin embargo, la versión que los autores suben al repositorio no es la versión final, conocida como versión del editor ( $\mathrm{VdE}$ ), sino un manuscrito que por lo general no ha sido debidamente editado, corregido orto-tipográficamente y maquetado por la editorial, y que con frecuencia tampoco incorpora las mejoras solicitadas por los pares. La VdE es retenida por la editorial durante un período de tiempo que suele durar entre 6 y 24 meses, lo que se conoce como período de embargo. La editorial intenta así conservar durante un tiempo prudencial el embargo para evitar perder suscripciones; es de suponer que, de estar las VdE de todos los artículos en abierto inmediatamente después de su publicación, las bibliotecas cancelarían las suscripciones. El interés por los artículos, al menos en las disciplinas pertenecientes a las ciencias llamadas "duras", decrece rápidamente en los primeros dos años de vida, por lo que el establecimiento de un período de embargo supone para las editoriales un factor determinante para proteger su inversión. Esta inversión no sólo corresponde a gastos de edición, corrección y maquetación, sino también, y sobre todo, a gastos de gestión del proceso de revisión por pares.

Ha habido (y todavía hay) algunos problemas relacionados con la vía verde (auto-archivo), como por ejemplo el miedo que algunos investigadores tienen a que no se les reconozca la prioridad de un determinado descubrimiento o el temor de estar violando derechos de propiedad editorial (Harnad, 2006, p. 79). Estas cuestiones están resueltas desde el punto de vista legal, pero muchos autores siguen siendo reticentes, por lo que es importante implementar una buen plan de comunicación cuando se inician proyectos que involucran repositorios digitales de acceso abierto, ya sean institucionales o temáticos.

La vía dorada, en cambio, aboga por un modelo más continuista: que los textos sean publicados por las revistas mismas en abierto, sin coste para los lectores. En este caso, para poder hacer frente a los costes de producción de artículos publicados en las revistas, las editoriales comerciales, sobre todo en el sector biomédico, han propuesto que sean los autores (o las instituciones a las que estos pertenecen) los que financien el libre acceso a los textos: el autor (o su institución) abona una cantidad para que la revista ofrezca gratuitamente el texto en internet. Este modelo se conoce como modelo del autor-paga y la cantidad que debe abonarse se conoce como article processing charge (APC), que corresponde a los costes de edición, corrección, maquetación y gestión de la revisión por pares.

\section{El gobierno del Reino Unido respaldó el Informe Finch, que recomendaba apoyar la vía de acceso abierto dorado}

La ventaja de este modelo es que permite que los artículos se publiquen en abierto inmediatamente, sin necesidad de un período de embargo. El inconveniente es que los autores o sus instituciones deben abonar una cantidad, a veces alta, que es evitable si se opta por la vía verde. Por ejemplo, revistas como Nature o editoriales como Kluwer o Springer piden US\$ 3.000 por artículo para permitir el acceso abierto.

Han surgido también otras críticas:

- los autores de países pobres o pertenecientes a disciplinas de orientación teórica con financiación reducida (como filosofía, literatura, matemáticas, física teórica, etc.) no pueden hacer frente a los costes de publicación OA;

- de tener que elegir, un investigador prefiere poder publicar gratis y pagar por leer que lo contrario, a saber, pagar por publicar y acceder a los artículos gratis.

El modelo que combina la publicación de artículos abiertos (tras el pago del APC) y artículos cerrados se conoce como OA híbrido.

Ante esta disyuntiva, el gobierno del Reino Unido encargó un informe (conocido como Informe Finch ${ }^{4}$ ) para identificar en el marco de la política científica de dicho país la mejor opción para implementar el OA a nivel nacional. El Informe Finch, publicado en junio de 2012, recomendaba priorizar la vía dorada ${ }^{5}$ frente a la verde y apoyarla económicamente a través de los planes nacionales de I+D del Reino Unido, empezando por el REF 2014-2020 . Para ello, proponía comenzar a aplicar las recomendaciones en abril de 2013 y destinar un presupuesto para apoyar económicamente a los investigadores que publicaran en revistas doradas. El gobierno británico dio su respaldo al informe un mes después y también promulgó el uso obligatorio de la licencia Creative Commons Attribution o de reconocimiento de la autoría 
(CC-BY). El uso de esta licencia no sólo permite el acceso abierto (gratuito), sino también la libre reutilización de los artículos, citando siempre la fuente. Los RCUK (Research Councils of $U K$ ) también apoyaron ${ }^{7}$ las recomendaciones del Informe Finch.

Como era de esperar, algunos autores relevantes que han defendido tradicionalmente la vía verde han criticado el Informe Finch (Harnad, 2012) pues consideran que ésta es la ruta más segura y rápida hacia un acceso abierto universal a la literatura científica. Stevan Harnad, máximo exponente e iniciador de la vía verde (Harnad, 2001), señala que el apoyo a la vía dorada es una victoria del lobby de las editoriales comerciales (Harnad, 2012) porque, además de los ingresos por la vía tradicional de las suscripciones, pueden obtener unos ingresos adicionales (vía autor-paga) que no se dan con la vía verde. Así, apuesta claramente por la vía verde y aboga por no financiar a las revistas doradas y obligar a los autores a depositar su artículos en repositorios. Otros autores de referencia, como por ejemplo Peter Suber (2012b) o Houghton y Swan (2013), abogan por impulsar ambas vías en paralelo.

Un informe ${ }^{8}$ publicado por el Business Innovation and Skills Committee de la House of Commons, del Parlamento Británico, el 3 de septiembre de 2013 señalaba como un error la decisión del Gobierno de centrarse casi exclusivamente en fomentar la vía dorada, al señalar que en los EUA y en la Unión Europea se estaba imponiendo la vía verde. Por ejemplo, universidades de prestigio mundial, tales como la Harvard University, u organismos financiadores de referencia, tales como el programa Horizonte 2020 lanzado por la Comisión Europea, obligan ya a los investigadores a depositar sus artículos en repositorios. Sin embargo, este informe, que al contrario que Harnad no se opone a la financiación de revistas doradas, sí insistía en que sólo deben financiarse las revistas doradas puras y no las híbridas, para evitar la doble financiación de dichas revistas, a saber, por la vía de las suscripciones y por la vía del autor-paga.

Una excepción a esta inclinación por el verde es Holanda, que en una declaración ${ }^{9}$ del secretario de estado de Educación, Cultura y Ciencia, mostraba su inclinación por la vía dorada. Es preciso señalar que tanto el Reino Unido como Holanda están a la cabeza de Europa en cuanto al número y tamaño de editoriales científicas comerciales, lo que ayuda a entender que sean más cautelosos a la hora de tomar decisiones que afecten o pongan en riesgo su industria editorial, tanto en materia de innovación como en materia de empleo.

Unos meses antes, en EUA, una directiva ${ }^{10}$ de la Casa Blanca (Administración Obama) publicada el 22 de febrero de 2013 hacía especial hincapié sobre la importancia que el acceso abierto a artículos y datos científicos tiene para el desarrollo tecnocientífico y socioeconómico del país:

"La investigación científica financiada por el Gobierno Federal cataliza avances innovadores que impulsan nuestra economía. Los resultados de esa investigación se convierten en la semilla para nuevas ideas y representan activos importantes para el progreso en áreas como la salud, la energía, el medio ambiente, la agricultura y la seguridad nacional.
El acceso a los conjuntos de datos digitales resultantes de la investigación financiada por el Gobierno Federal permite a las empresas focalizar los recursos y los esfuerzos en la comprensión y explotación de los descubrimientos. Por ejemplo, los datos en abierto sobre el clima refuerzan la industria de previsiones meteorológicas, y poner la secuenciación del genoma a disposición del público ha dado lugar a numerosas innovaciones biotecnológicas. Además, una mayor accesibilidad a las publicaciones revisadas por pares y a los datos científicos en formatos digitales creará mercados económicos innovadores para los servicios relacionados con la conservación, la preservación, el análisis y la visualización. Las políticas que permiten que estas publicaciones y estos datos puedan ser reutilizados a través de la preservación y el amplio acceso público también maximizarán el impacto y la visibilidad de la rendición de cuentas de la inversión federal en investigación. Estas políticas acelerarán los avances científicos y la innovación, fomentarán el espíritu empresarial y mejorarán el crecimiento económico y la creación de empleo".

Y solicitaba a todas las agencias financiadoras de EUA que promulgaran la obligatoriedad de publicar en abierto todos los artículos financiados con fondos públicos. En particular, la directiva no hacía mención expresa a financiar la publicación en revistas doradas, pero tampoco descartaba esta opción. Sin embargo, sí señalaba (pág. 3, apartado 3.a.i.) que el período de embargo de las revistas no debía superar los 12 meses, salvo contadas excepciones debidamente justificadas (pág. 3, apartado 3.a.ii.). Y añadía que, independientemente del tiempo de embargo, los investigadores debían depositar sus postprints en repositorios institucionales o temáticos inmediatamente después de ser aceptados por las revistas, para poner a disposición de las personas y de las máquinas los metadatos de los artículos (pág. 3, apartado 3.c.).

Harnad señala que el apoyo al camino de acceso abierto dorado es una victoria del lobby de las editoriales comerciales

En octubre de 2013, fue publicado un segundo Informe Finch $^{11}$. Tras las críticas recibidas y probablemente influido por la directiva estadounidense, el Informe Finch II era más imparcial en relación con las dos vías posibles. Además del apoyo financiero a la vía dorada, recomendaba que las instituciones financiadoras implementaran la obligatoriedad de depositar los artículos en los repositorios institucionales inmediatamente después de que la revista correspondiente aceptara publicar el artículo, el mismo criterio que la directiva de la Administración Obama. En otras palabras, los artículos estarían depositados inmediatamente en los repositorios, pero no estarían accesibles en abierto hasta después de transcurrido el tiempo de embargo. No obstante, el Informe insistía en que la decisión final en la elección entre dorado y verde debía dejarse al criterio de los autores de los artículos. Así, si los autores se inclinaban por revistas doradas, debía implementarse un mecanismo para financiar esta opción. 
Open access to 1,064,100 e-prints in Physics, Mathematics, Computer Science, Quantitative Biology, Quantitative Finance and Statistics

Subject search and browse: Physics Search Form Interface Catchup

12 Jan 2015: Reflections on the 1 million paper milestone

12 Jan 2015: A project update, including a brief summary of activities in 2014, has been posted

1 Jan 2015: New members join arXiv Scientific Advisory Board

See cumulative "What's New" pages. Read robots beware before attempting any automated download

http://arxiv.org

El objetivo del presente artículo es descubrir el origen histórico de la bifurcación del OA en dos caminos, el dorado y el verde, y explicar los motivos que han llevado al Informe Finch a inclinarse por la vía dorada. El análisis mostrará que el lobby que está a favor de la vía dorada tiene sus orígenes en las políticas de publicación de la revista The New England journal of medicine: su director, Franz J. Ingelfinger, introdujo una norma de publicación que con el tiempo se ha dado en Ilamar la regla de Ingelfinger. Esta norma apelaba a la originalidad de los manuscritos y a la prohibición de divulgar información a los medios de comunicación antes de publicar el artículo en su revista.

\section{Antecedentes}

El origen del movimiento OA se remonta a 1991. En julio de aquel año un grupo de matemáticos, Hans Koch, Rafael De la Llave y Charles Radin, crearon en la University of Texas (Austin) un servidor de ficheros, llamado mp_arc (mathematical-physics preprint archive), con el propósito de acelerar el intercambio (de borradores) de artículos aún no formalmente aceptados para publicar (preprints). Un mes más tarde, en agosto, el físico teórico de altas energías Paul Ginsparg creó el hep_th (high energy physics-theoretical) en Los Alamos National Laboratory.

Mientras que el $m p \_a r c$ se quedó en un archivo de unos 2.500 preprints y 850 suscriptores, hep_th se convirtió con el tiempo en $\operatorname{arXiv.org}^{12}$, un repositorio digital que contiene más de un millón de preprints ${ }^{13}$.

Financiado por la National Science Foundation (NSF) y el Departamento de Energía del Gobierno de EUA (Kling; Spector; McKim, 2002) entre otras instituciones, el repositorio arXiv -alojado en la Cornell University desde 2001- se convirtió en el ciber-lugar o e-agora en el que los físicos depositaban sus textos inmediatamente antes de enviarlos a las correspondientes revistas con el fin de publicar por los canales formales. Aprovechando la irrupción de internet, el objetivo de estos pioneros era alcanzar la inmediatez en el intercambio de la información, esto es, poner los descubrimientos a disposición de los pares en el menor tiempo posible, sin tener que esperar los meses de rigor característicos de las revistas académicas impresas. La gran novedad de arXiv era que permitía (y permite) el acceso gratuito y universal al contenido completo de los preprints.
Tras el éxito logrado, el ejemplo de arXiv fue tomado muy en serio en 1998 por varias instituciones relacionadas con la biomedicina. Primero despertó el interés de David Lipman, director del National Center of Biotechnology Information (NCBI), y de Pat Brown, investigador de la Stanford University, que recomendaron la creación de un repositorio similar a arXiv en biomedicina. Propusieron Ilamarlo E-Biomed. Poco tiempo después se unió a la iniciativa Harold Varmus, director de los National Institutes of Health (NIH), la agencia gubernamental responsable de la investigación biomédica en EUA. Varmus se comprometió a financiar el proyecto con fondos de los $\mathrm{NIH}$. El objetivo era que E-Biomed tuviera dos secciones:

- un servidor de preprints tipo arXiv;

- un repositorio con artículos revisados por los pares y ya publicados en revistas (postprints).

Al igual que arXiv, estaba previsto que el acceso a E-Biomed fuera gratuito y que los propios investigadores fueran los responsables de depositar (auto-archivar) sus artículos, fueran éstos preprints o postprints. La ventaja del servidor de preprints sobre el de postprints radicaba en que el primero permitía el intercambio inmediato de información sin necesidad de esperar a la revisión por pares. En otras palabras, mientras que el repositorio de preprints era sinónimo de acceso abierto inmediato, el servidor de postprints implicaba un acceso abierto diferido.

A pesar de unos comienzos esperanzadores, la iniciativa pronto quedó marginada a una alternativa más modesta, PubMed Central, que no incluía la sección de preprints. Fueron sobre todo las editoriales comerciales tradicionales en el campo de la biomedicina (además de la revista Proceedings of the National Academy of Sciences, PNAS) y las sociedades científicas, tales como la American Society for Cell Biology y la American Society of Plant Physiologists, las que ejercieron presión para impedir la creación del servidor de preprints en E-Biomed. Por el contrario, la inmensa mayoría de investigadores se mostraron en todo momento partidarios del acceso abierto a los preprints.

A principios de 1999, Varmus envió la propuesta de crear E-Biomed a PNAS, la prestigiosa revista interdisciplinar de la National Academy of Sciences (NAS). Sin embargo, lo que parecía ser un puro trámite de gobernanza científica se con- 
virtió en el mayor obstáculo para la creación de E-Biomed. El comité editorial de PNAS rechazó apoyar la iniciativa si se mantenía la sección dedicada al servidor de preprints. En opinión de Kling et al. (2001), este rechazo por parte de PNAS fue un revés político clave que determinaría la conversión de E-Biomed en PubMed Central.

La iniciativa PubMed Central se convertía así en una versión light de la idea original. Se eliminaba el auto-archivo de preprints por parte de los autores/investigadores y se creaba un Comité Asesor encargado de vigilar las nuevas "reglas" de publicación de postprints en el repositorio. En adelante, sólo las editoriales, las sociedades científicas y otros grupos concertados tendrían permiso para publicar artículos en PubMed Central. Esto garantizaba la publicación únicamente de artículos que hubieran pasado algún tipo de filtro formal en el seno de cada uno de estos grupos, por ejemplo un proceso de revisión por pares. Además, con el fin de proteger su negocio, los grupos editoriales se reservaban el derecho a depositar (en abierto) en PubMed Central, con seis meses o un año de retraso, los artículos publicados originalmente en sus revistas, es decir, un embargo temporal.

\section{Tras el éxito logrado, el ejemplo de arXiv fue tomado muy en serio en 1998 por varias instituciones relacionadas con la biomedicina}

¿Cuales fueron las razones que provocaron que, a pesar del entusiasmo inicial de los investigadores y de varias instituciones como los NIH, el deseado "arXiv de los médicos" no fuera tal? Hay varias respuestas posibles. Por un lado, hay que señalar que eran muchos los actores cuyos intereses, económicos o de otro tipo, podían verse gravemente amenazados por la decisión. El sector de las publicaciones biomédicas es una pequeña parte de un complejo tecnocientífico de mayores proporciones: la suma de la industrias biomédica y farmacéutica. La irrupción de la inmediatez y de la gratuidad gracias al soporte telemático implicaba un aumento en la incertidumbre y la emergencia de nuevas amenazas, tanto para las editoriales comerciales como para las revistas pertenecientes a sociedades científicas. Y la publicación de ensayos clínicos o de nuevos fármacos podía poner en riesgo patentes e inversiones.

El perjuicio económico que el OA a los artículos científicos podía suponer para las revistas comerciales era obvio. En primer lugar, la gratui- dad de los servidores de preprints ponía en riesgo la política de suscripciones de las bibliotecas, es decir, las bibliotecas podían interpretar que no merecía la pena pagar grandes sumas de dinero en suscripciones por contenidos depositados en dichos repositorios abiertos. Las bibliotecas podían entonces presionar los precios a la baja o incluso cancelar suscripciones. En segundo lugar, los ingresos por publicidad podían verse gravemente mermados si los anunciantes, por ejemplo las empresas farmacéuticas, decidían retirarse en respuesta a la potencial disminución de lectores. Y en tercer lugar, los repositorios amenazaban con destruir el concepto de Factor de Impacto tradicional basado en el prestigio de las revistas. En otras palabras, si se daba el caso que los investigadores empezaran a adquirir la costumbre de citar directamente los artículos depositados en los repositorios en lugar de citar aquellos que fueran publicados en las revistas, el factor de impacto de dichas revistas podía verse afectado, con las consiguientes pérdidas de prestigio y disminución en las ventas de suscripciones. Por tanto, con el fin de salvaguardar el prestigio de las revistas y hacer ver a la comunidad biomédica la necesidad de mantener un procedimiento que garantizara la calidad de los contenidos publicados, las revistas comerciales debían persuadir a toda costa a la comunidad biomédica de las bondades del proceso de revisión por pares. Sin embargo, es preciso señalar que no todas las revistas comerciales se opusieron al servidor de preprints de E-Biomed. Por ejemplo, la prestigiosa revista The lancet apoyó la propuesta, mientras que The New England journal of medicine (NEJM) y el Journal of the American Medical Association (JAMA), entre otras, se mostraron radicalmente en contra. Más adelante, al analizar el caso de la NEJM, veremos la enorme influencia de la política editorial de esta revista, practicada desde el año 1969, sobre el desenlace de la iniciativa de E-Biomed.

En cuanto a las revistas pertenecientes a sociedades y asociaciones científicas, como puede ser el caso de PNAS, la cosa es más complicada. A pesar de que estas sociedades y asociaciones no tienen ánimo de lucro, no es menos cierto que parte de sus ingresos provienen de la venta de la revis-

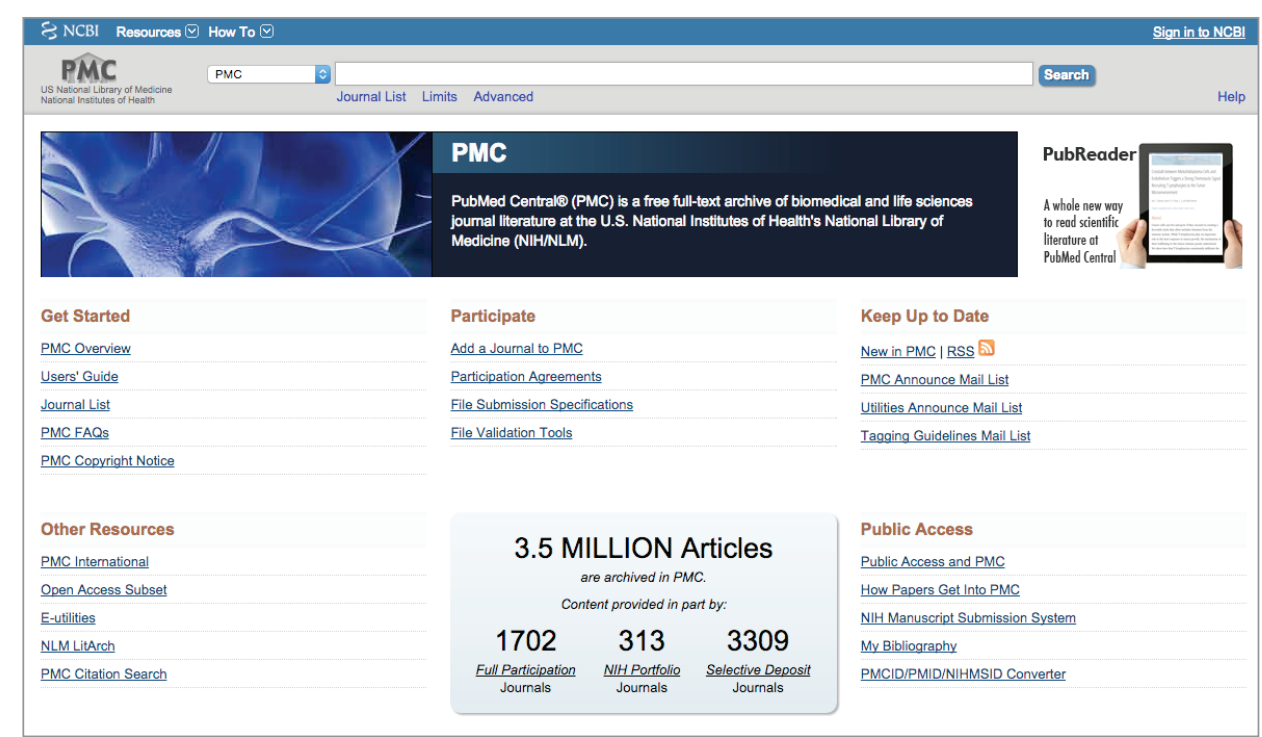

http://www.ncbi.nlm.nih.gov/pmc 
ta asociada. Estos ingresos, que se añaden a las cuotas que aportan sus miembros, permiten a estas sociedades ofrecer becas remuneradas, convocar y entregar premios, encargar informes científicos en sus respectivas áreas de estudio con el propósito de influir social y políticamente en las voluntades ciudadanas y en las decisiones gubernamentales, respectivamente, etc. Los beneficios económicos que estas sociedades extraían (y extraen) de la venta de suscripciones son significativas $y$, aunque no tienen detrás la presión de unos accionistas, es innegable que redundaba en su interés y en el de su disciplina mantener el status quo. Un caso conocido es el de la Royal Society de Londres (2005), que se mostró especialmente vehemente en contra del movimiento $\mathrm{OA}$.

EUA solicitaba a todas las agencias financiadoras de su país que promulgaran la obligatoriedad de publicar en OA todos los artículos financiados con fondos públicos

\section{La larga sombra de Ingelfinger}

En 1969, Franz J. Ingelfinger, editor de NEJM, promulgó una norma a la que debían someterse en adelante todos aquellos autores que desearan publicar un artículo en su revista. Esta norma, conocida como Ingelfinger rule (o regla de Ingelfinger), establecía que su revista sólo aceptaría revisar artículos cuyas ideas principales no hubieran sido divulgadas antes por otros medios: "entendemos que el material enviado a la Revista [NEJM] no ha sido ofrecido a ningún libro, revista o periódico" (Ingelfinger, 1969).

Ingelfinger señaló que su objetivo era impedir que los autores publicaran en su revista contenidos que no fueran originales con el fin de evitar, entre otras cosas, la creciente mala costumbre de publicar textos redundantes. El incremento de los gastos en I+D tras la segunda guerra mundial, financiada en su mayor parte con fondos públicos y especialmente relevante en biomedicina (Willinsky, 2005, p. 14), motivó un mayor control de la productividad investigadora por parte de los gobiernos, vía factor de impacto u otros indicadores similares, y provocó que la evaluación por pares se tomara como vara de medir para garantizar (en lo posible) no sólo la producción de buena ciencia, sino también "que los impuestos de los contribuyentes destinados a la ciencia y a la medicina sean distribuidos de manera justa y efectiva" (Biagioli, 2002, p. 13). Todas estas medidas empujaron a muchos científicos a publicar en exceso en un clima negativo de publish or perish. Esta presión por publicar ha impulsado la práctica de duplicar y fragmentar la difusión de la información mediante la técnica conocida como salami slicing (Nature, 2005), es decir, contar en muchos artículos lo que podía perfectamente decirse en uno.

Sin embargo, la motivación de fondo de Ingelfinger era sobre todo comercial (Altman, 1996a): evitar que los medios de comunicación de masas y otras revistas publicaran las primicias biomédicas antes que NEJM. Perder la primicia informativa significaba perder lectores: "si la Revista [NEJM] publicara sólo aquello que es ya sabido, que está confirmado y que está listo para ser incorporado a los libros de texto en lugar de intentar tener a los médicos actualizados con la información más reciente, entonces nuestra revista sufriría una disminución inevitable en importancia, en prestigio y en número de lectores" (Ingelfinger, 1977).

Y perder lectores implicaba, por extensión, perder citas potenciales y recibir menos ofertas publicitarias para anunciar productos farmacéuticos (Altman, 1996b). Tras poner en práctica la norma, los beneficios económicos netos de NEJM aumentaron espectacularmente entre 1970 y 1979, pasando de 93.257 a 386.540 US\$ (Altman, 1996b).

Con el propósito de disimular las motivaciones económicas y recalcar las virtudes éticas de la norma, el sucesor de Ingelfinger, A. S. Relman (1979), hizo entonces hincapié en el proceso de revisión por pares como garantía para evitar que el público o los médicos de cabecera se hicieran eco de información biomédica falsa, inexacta, incompleta o aún no debidamente contrastada. Relman hacía un llamamiento a la prudencia al afirmar que cualquier información biomédica debía ser revisada por los pares (esto es, un juicio epistémico previo) antes de ser publicada en abierto:

"Creemos que la investigación médica debe ser sometida a revisión por pares y publicada en la literatura científica antes de ser difundida al público o a los profesionales [de la medicina]" (Relman, 1981).

Relman (1980) hizo extensiva a los congresos científicos la norma de Ingelfinger de no proporcionar información con anterioridad a su publicación en NEJM:

“Me parece que la lección para las sociedades de investigación es que los organizadores de reuniones científicas deben pensárselo dos veces antes de alentar a los medios de comunicación a que difundan las conclusiones preliminares presentadas en dichas reuniones".

Con la aparición de internet, los editores que sucedieron a Ingelfinger y a Relman extremaron aún más la vigilancia. Así, los servidores de preprints se convirtieron en el nuevo frente a combatir por parte de J. P. Kassirer y M. Angell, nuevos coeditores de $N E J M$, sobre todo al ver el éxito al que ya apuntaba el repositorio arXiv en los campos de la física y las matemáticas:

“El acceso público a los preprints de estudios médicos colgados en internet podría llevar a algunas personas a usar un medicamento equivocado o a dejar de tomar otro necesario sobre la base de información inadecuada. [...] La comunicación electrónica puede convertirse en algo muy importante para la medicina. [...] Pero la medicina no es como la física: es poco probable que la amplia circulación de preprints inéditos en física tenga un efecto inmediato en el bienestar del público, incluso si su contenido está sesgado o es falso. En medicina, esta práctica podría tener consecuencias no intencionadas que podríamos posteriormente lamentar" (Kassirer; Angell, 1995).

La norma de Ingelfinger se convirtió en un llamamiento a la prudencia como el valor ético a priorizar, por encima del va- 
lor ético asociado a la libertad de difundir el conocimiento.

Declaraciones más recientes de editores de revistas comerciales han seguido la estela marcada por Kassirer y Angell. Por ejemplo, el director de Wiley Europe, John Jarvis, afirmaba en 2004 que el OA conducía irremediablemente al caos, y añadía:

"Pregunten a la gente que pertenece a la profesión médica, y les responderán que la última cosa que desean es gente con enfermedades leyendo estas informaciones [de internet], discutiendo sobre cirugías y pidiendo cosas" (citado en Willinsky, 2005, p. 8).

Pero, ¿'hasta qué punto contaban los valores éticos para NEJM? Lindsay Waters, editor ejecutivo en humanidades de Harvard University Press, nos ofrece una pista sobre esta cuestión al escribir que:

"el editor del New England Journal of Medicine, Jerome Kassirer, se vio apartado en 1999 [siendo sustituido por M. Angell] porque los dueños no estaban contentos con la posición ética que tomó para asegurar la calidad de la revista. Antepuso la calidad sobre los ingresos. Los dueños querían que actuara de manera más "emprendedora" [...]". (Waters, 2004, p. 23).

La norma de Ingelfinger se convirtió en un llamamiento a la prudencia como el valor ético a priorizar, por encima del valor ético asociado a la libertad de difundir el conocimiento

\section{Conclusiones}

Son muchos los agentes y los intereses que rodean a la investigación científica. La imagen epistémica de la ciencia, basada en la ingenua creencia de que la ciencia es cosa sólo de científicos, ha quedado superada. La ciencia se ha convertido en una industria. La utilización por parte de la científicos de tecnologías y de instrumentos cada vez más avanzados, el interés creciente del público por los resultados de las investigaciones científicas y por la eficiente gestión del erario público dedicado a la I+D, y el creciente impacto de ésta en la calidad de vida de los ciudadanos, han convertido a la ciencia en un objeto con muchos sujetos. Por supuesto, los científicos siguen siendo parte esencial de la actividad científica, pero han dejado de ser los únicos que influyen decisivamente en su desarrollo. Empresas farmacéuticas, ingenieros, editores de revistas, bibliotecarios, oficinas de patentes, doctorandos, gestores de I+D y periodistas científicos, entre otros, conforman también la nueva imagen de la ciencia: un amplio abanico de agentes, en ocasiones con intereses contrapuestos y valores en conflicto, que negocian espacios de poder en el seno de una ciencia cada vez más tecnificada y de carácter más utilitarista. Estamos pues hablando de tecnociencia en sentido amplio (Echeverría, 2003), es decir, como un área cada vez más tecnificada en la que interactúan muchos agentes movidos por diversos intereses y valores.
En la actualidad, la biomedicina tiene un impacto social mayor que la física, ya sea ésta teórica o experimental. Y el número de agentes y de valores que rodean la práctica biomédica también son mayores en el área biomédica. Por ejemplo, la expectación social que rodea la aparición de nuevos fármacos que mitiguen o eliminen ciertas dolencias y enfermedades es probablemente mayor que el avistamiento de un nuevo planeta o el descubrimiento de un teorema algebraico. Por ello, el interés periodístico por (y la utilización política de) las noticias y los resultados de las investigaciones biomédicas es también superior. Véase por ejemplo el contagio de ébola en España en octubre 2014, con una repercusión mediática y con consecuencias sociales y políticas (no sólo médicas), de gran calado ${ }^{14}$.

\section{El camino hacia un acceso abierto más} laxo es el resultado de mayor pluralidad axiológica de la biomedicina

Al igual que ocurrió con los físicos, la presión ejercida por investigadores y bibliotecarios obligó a la comunidad biomédica a emprender el camino hacia el OA. Los investigadores anhelaban la inmediatez en el intercambio de artículos, mientras que los bibliotecarios perseguían frenar el alza de precios de las suscripciones. Sin embargo, el OA ha seguido caminos diferentes en función de según qué disciplinas: lo físicos implementaron el camino verde sin dificultad; mientras que en biomedicina, como hemos visto, las fuerzas ejercidas por diversos agentes con diversos intereses y valores han desembocado en presiones por un OA más laxo y de menor impacto sobre la forma tradicional de comunicación académica: el camino dorado. La conclusión es que esta divergencia, y mayor resistencia al OA por parte de las revistas biomédicas, tiene su explicación en la mayor pluralidad axiológica de la biomedicina en relación a otras disciplinas tales como la física de partículas o la geometría.

\section{Agradecimientos}

El presente artículo forma parte del proyecto CSO201347767-C2-1-R: Big data, redes sociales y periodismo de datos: aplicación de las herramientas de monitorización al análisis de fuentes y contenidos periodísticos, financiado por el Ministerio de Economía y Competitividad.

\section{Notas}

1. Información completa y detallada sobre OA disponible en la web de Peter Suber:

http://www.earlham.edu/ peters

2. The Budapest open access initiative (14 febrero 2002), patrocinado por el Open Society Institute:

http://www.budapestopenaccessinitiative.org/read

Y el Bethesda statement on open access publishing (20 junio 2003):

http://dash.harvard.edu/bitstream/handle/1/4725199/ suber_bethesda.htm?sequence $=1$

3. Berlin declaration on open access to knowledge in the sciences and humanities (22 octubre 2003), 
http://oa.mpg.de/lang/en-uk/berlin-prozess/berliner-erklarung

4. Report of the Working Group on Expanding Access to Published Research Findings, "Accessibility, sustainability, excellence: how to expand access to research publications". http://www.researchinfonet.org/wp-content/uploads/2012/06/ Finch-Group-report-FINAL-VERSION.pdf

5. Otra fuente de recomendaciones puede verse en: http://www.budapestopenaccessinitiative.org/boai-10recommendations

6. El REF (Research excellence framework) es una convocatoria del Hefce (Higher Education Funding Council for England).

7. RCUK announces new open access policy http://www.rcuk.ac.uk/media/news/120716

8. Business, Innovation and Skills Committee - Fifth report, Open access,

http://www.publications.parliament.uk/pa/cm201314/ cmselect/cmbis/99/9902.htm

9. http://www.government.nl/documents-and-publications/ parliamentary-documents/2014/01/21/open-access-topublications.html

10. http://www.whitehouse.gov/sites/default/files/microsites/ ostp/ostp_public_access_memo_2013.pdf

(traducido al español por el autor del presente artículo).

11. A review of progress in implementing the recommendations of the Finch Report, "Accessibility, sustainability, excellence: how to expand access to research publications", http://www.researchinfonet.org/wp-content/uploads/2013/02/ Final-version.pdf

\section{2. http://arxiv.org}

13. A fecha 4 de abril de 2015 .

14. http://www.elmundo.es/espana/2014/10/10/543723b bca4741bf438b4590.htm/?a=b8c7243fc6b488af9fbf994fc5 e37252\&t=1412934955

\section{Bibliografía}

Altman, Lawrence K. (1996a). "The Ingelfinger rule, embargoes, and journal peer review - Part 1". The lancet, v. 347, n. 9012, pp. 1382-1386.

http://dx.doi.org/10.1016/S0140-6736(96)91016-8

Altman, Lawrence K. (1996b). "The Ingelfinger rule, embargoes, and journal peer review - Part 2". The lancet, v. 347, n. 9013, pp. 1459-1463.

http://dx.doi.org/10.1016/S0140-6736(96)91689-X

Biagioli, Mario (2000). "Rights or rewards: Changing contexts and definitions of scientific authorship". Journal of college and university law, v. 27, n. 1, pp. 83-108.

http://innovation.ucdavis.edu/people/publications/ Biagioli_Rights_Rewards.pdf

Biagioli, Mario (2002). "From book censorship to academic peer review". Emergences: Journal for the study of media and composite cultures, v. 12, n. 1, pp. 11-45.

http://innovation.ucdavis.edu/people/publications/ Biagioli\%202008\%20Censorship_review.pdf http://dx.doi.org/10.1080/1045722022000003435

Echeverría, Javier (2003). La revolución tecnocientífica. Fondo de Cultura Económica de España. ISBN: 978 8437505510

Ginsparg, Paul (2007). "Next-generation implications of open access". CTWatch quarterly, v. 3, n. 3.

http://www.ctwatch.org/quarterly/articles/2007/08/nextgeneration-implications-of-open-access

Guédon, Jean-Claude (2001). In Oldenburg's long shadow: Librarians, research scientists, publishers, and the control of scientific publishing. Washington: Association of Research Libraries. ISBN: 0918006813

http://www.arl.org/resources/pubs/mmproceedings/138guedon. shtml

Harnad, Stevan (2001). "The self-archiving initiative". Nature, n. 410, pp. 1024-1025.

http://www.nature.com/nature/debates/e-access/Articles/ harnad.html

Harnad, Stevan (2003). "Electronic preprints and postprints". Encyclopedia of library and information science. Marcel Dekker, Inc.

http://users.ecs.soton.ac.uk/harnad/Temp/eprints.htm

Harnad, Stevan (2012). "Why the UK should not heed the Finch Report". LSE impact of social sciences blog (summer issue), July 4.

http://eprints.soton.ac.uk/341128

Houghton, John; Swan, Alma (2013). "Planting the green seeds for a golden harvest: Comments and clarifications on 'going for gold'”. D-Lib magazine, v. 19, n. 1/2, January/February. http://dx.doi.org/10.1045/january2013-houghton

Ingelfinger, Franz J. (1969). "Definition of 'sole contribution'", The New England journal of medicine, n. 281, pp. 676-677.

http://dx.doi.org/10.1056/NEJM196909182811208

Ingelfinger, Franz J. (1977). "The general medical journal: for readers or repositories?". The New England journal of medicine, n. 296, pp. 1258-1264.

http://dx.doi.org/10.1056/NEJM197706022962204

Kassirer, Jerome P.; Angell, Marcia (1995). "The internet and the journal". The New England journal of medicine, $\mathrm{n}$. 332, pp. 1709-1710.

http://dx.doi.org/10.1056/NEJM199506223322509

Kling, Rob; Spector, Lisa; Fortuna, Joanna (2004). "The real stakes of virtual publishing: The transformation of E-Biomed into PubMed Central". Journal of the American Society for Information Science and Technology, v. 55, n. 2, pp. 127-148. http://dx.doi.org/10.1002/asi.10352

Kling, Rob; Spector, Lisa; McKim, Geoff (2002). "Locally controlled scholarly publishing via the internet: The guild model". The journal of electronic publishing, v. 8, n. 1. http://dx.doi.org/10.3998/3336451.0008.101

Nature (2005). "The cost of salami slicing". Editorial, Nature materials, v. 4, n. 1.

http://dx.doi.org/10.1038/nmat1305 
Relman, Arnold S. (1979). "An open letter to the news media”. The New England journal of medicine, n. 300, pp. 554-555. http://dx.doi.org/10.1056/NEJM197903083001009

Relman, Arnold S. (1981). "The Ingelfinger rule". The New England journal of medicine, n. 305, pp. 824-826.

http://dx.doi.org/10.1056/NEJM198110013051408

Royal Society (2005). "Royal Society warns hasty 'open access' moves may damage science".

http://www.royalsoc.ac.uk/news.asp?id=3881

Rusnock, Andrea (1999). "Correspondence networks and the Royal Society, 1700-1750". British journal for the history of science, v. 32, n. 2, pp. 155-169.

Suber, Peter (2012a). Open access. Cambridge, MA: The MIT Press. ISBN: 9780262517638 https://mitpress.mit.edu/sites/default/files/9780262517638 Open_Access_PDF_Version.pdf

Suber, Peter (2012b). "Ensuring open access for publicly funded research". British medical journal, n. 345, e5184, http://dx.doi.org/10.1136/bmj.e5184

Till, James E. (2001). "Predecessors of preprint servers". Learned publishing, v. 14, n. 1, pp. 7-13.

http://arxiv.org/html/physics/0102004

http://dx.doi.org/10.1087/09531510125100214

Waters, Lindsay (2004). Enemies of promise: Publishing, perishing, and the eclipse of scholarship. Chicago: Prickly Paradigm Press. ISBN: 9780972819657

Willinsky, John (2005). The access principle: The case for open access to research and scholarship. Cambridge, MA: The MIT Press. ISBN: 0262232421



